\title{
Characteristic Evaluation on Bolt Stress by Ultrasonic Nondestructive Methods
}

\author{
Qinxue Pan, ${ }^{1,2}$ Shuai Liu, ${ }^{1}$ Xiao $\mathrm{Li}^{3}$ and Chunguang $\mathrm{Xu}^{1,2}$ \\ ${ }^{1}$ School of Mechanical Engineering, Beijing Institute of Technology, Beijing 100081, China \\ ${ }^{2}$ Key Laboratory of Fundamental Science for National Defense for Advanced Machining Technology, Beijing 100081, China \\ ${ }^{3}$ BMW China Services Ltd., Beijing 101312, China \\ Correspondence should be addressed to Qinxue Pan; panqx@bit.edu.cn
}

Received 15 May 2015; Revised 9 July 2015; Accepted 26 July 2015

Academic Editor: Rui Vilar

Copyright (C) 2015 Qinxue Pan et al. This is an open access article distributed under the Creative Commons Attribution License, which permits unrestricted use, distribution, and reproduction in any medium, provided the original work is properly cited.

Based on the acoustoelasticity theory, a certain relationship exists between ultrasonic velocity and stress. By combining shear and longitudinal waves, this paper provides a nondestructive method of evaluating axial stress in a tightened bolt. For measuring the bolt axial stress in different situations, such as under low or high loads, this paper provides guidelines for calculating the stress for a given load factor. Experimental and calculated results were compared for three bolt test samples: an austenitic stainless steel bolt (A2-70) and low-carbon steel 4.8 and 8.8 bolts. On average, the experimental results were in good agreement with those obtained through calculations, thus providing a nondestructive method for bolt stress measurements.

\section{Introduction}

Bolts, a key component in equipment joints, are used to join, clamp, reinforce, and seal various parts and are widely used throughout industry (e.g., aerospace, marine, construction, chemical, and energy industries). Due to their importance, bolt axial stress has received much attention in an attempt to improve the bolt's performance and useful life. However, the complexities of the bolt structure and threaded part deformation with axial loading have prevented accurate measurement of the axial stress. In recent decades, the research community has actively explored solutions to this problem [1]. Williams et al. gave the ultrasonic analysis of bolt preload [2], Johnson et al. used time-of-flight measurements of both shear and longitudinal waves propagating along the axis of a bolt along with the material parameters to calculate the stress acting on the bolt [3], and Koshti developed the application to make ultrasonic measurement of preload in sleeve bolts [4]. At present, a torque wrench is commonly used to determine the degree of bolt axial stress in the industrial field. However, due to the reverse torque in the nut of the thread friction pair, as well as the elastic deformation, the torque used to produce the bolt axial stress is actually $<20 \%$ of the torque wrench numerical value [5]. Ultrasonic nondestructive testing of the vertical screw bolt axial stress (e.g., the pulse echo reflection method, transit time method, and phase method) cannot measure the axial stress when the bolts are installed or buried to a depth at which the original length of the anchor bolt is unknown $[6,7]$.

Ultrasonic nondestructive testing with electromagnetic ultrasonic shock excitation shear waves has been used to measure the axial pretightening force of bolts, with some promising results; however, this approach fails to resolve the axial stress measurement when the bolt length is unknown [8]. According to the acoustoelasticity theory, the ultrasonic velocity and the magnitude of the stress in isotropic metal materials are related in such a way that the effect of the bolt axial length and temperature change are eliminated through simultaneous solving of the transverse and longitudinal wave equations [9-13]. However, in practice, the relationship between the acoustic time difference and the stress magnitude does not have a simple linear form under extremely low or high loading conditions. Thus, a more detailed stress measurement model is required to accurately resolve the bolt axial stress under various loading conditions. 


\section{Theory}

From the sound theory of elasticity, the elastic wave propagation velocity in a solid material, the material itself (e.g., material density), and the second-order elastic constants are closely related to the stress state of the material. As such, the stress medium elastic wave equation for the initial coordinate system can be expressed as follows:

$$
\frac{\partial}{\partial X_{J}}\left[\left(\delta_{I K} t_{J L}^{i}+C_{I J K L}\right) \frac{\partial u_{K}}{\partial X_{L}}\right]=\rho^{i} \frac{\partial^{2} u_{i}}{\partial t^{2}},
$$

where $\delta_{I K}$ is a parameter of the Kronecker delta function, $\rho^{i}$ is the material density, $u_{i}$ is the distance of propagation, $X_{J}$ is the vector particle of a point, $C_{I J K L}$ is the equivalent stiffness, and $t_{J L}^{i}$ is the Cauchy stress.

Elastic sound is nonlinear. Assuming a plane ultrasound wave and the initial coordinates, (1) can be simplified to the following form:

$$
u_{i}=U_{I} \exp \left[i k\left(N_{J} X_{J}-V t\right)\right]
$$

Taking into account the stress state in which the speed of sound and the elastic waves in the material have a quantitative mathematical relationship, and given the independent Lagrange variables $a, b$, and $c$, and the particle displacement described by $u, v$, and $w$, we have the following:

$$
\begin{aligned}
& \rho_{0} \frac{\partial_{u}^{2}}{\partial_{t}^{2}}=\frac{\partial T_{11}}{\partial a}+\frac{\partial T_{12}}{\partial b}+\frac{\partial T_{13}}{\partial c}, \\
& \rho_{0} \frac{\partial_{v}^{2}}{\partial_{t}^{2}}=\frac{\partial T_{21}}{\partial a}+\frac{\partial T_{22}}{\partial b}+\frac{\partial T_{23}}{\partial c}, \\
& \rho_{0} \frac{\partial_{w}^{2}}{\partial_{t}^{2}}=\frac{\partial T_{31}}{\partial a}+\frac{\partial T_{32}}{\partial b}+\frac{\partial T_{33}}{\partial c} .
\end{aligned}
$$

The stress $T_{i j}$ in (3) is given below:

$$
\begin{aligned}
& T_{11}=J_{11} \frac{\partial \phi}{\partial \eta_{11}}+J_{12} \frac{\partial \phi}{\partial \eta_{21}}+J_{13} \frac{\partial \phi}{\partial \eta_{31}}, \\
& T_{12}=J_{11} \frac{\partial \phi}{\partial \eta_{12}}+J_{12} \frac{\partial \phi}{\partial \eta_{22}}+J_{13} \frac{\partial \phi}{\partial \eta_{32}}, \\
& T_{13}=J_{11} \frac{\partial \phi}{\partial \eta_{13}}+J_{12} \frac{\partial \phi}{\partial \eta_{23}}+J_{13} \frac{\partial \phi}{\partial \eta_{33}}, \\
& T_{21}=J_{21} \frac{\partial \phi}{\partial \eta_{11}}+J_{22} \frac{\partial \phi}{\partial \eta_{21}}+J_{23} \frac{\partial \phi}{\partial \eta_{31}}, \\
& T_{22}=J_{21} \frac{\partial \phi}{\partial \eta_{12}}+J_{22} \frac{\partial \phi}{\partial \eta_{22}}+J_{23} \frac{\partial \phi}{\partial \eta_{32}},
\end{aligned}
$$

$$
\begin{aligned}
& T_{23}=J_{21} \frac{\partial \phi}{\partial \eta_{13}}+J_{22} \frac{\partial \phi}{\partial \eta_{23}}+J_{23} \frac{\partial \phi}{\partial \eta_{33}} \\
& T_{31}=J_{31} \frac{\partial \phi}{\partial \eta_{11}}+J_{32} \frac{\partial \phi}{\partial \eta_{21}}+J_{33} \frac{\partial \phi}{\partial \eta_{31}} \\
& T_{32}=J_{31} \frac{\partial \phi}{\partial \eta_{12}}+J_{32} \frac{\partial \phi}{\partial \eta_{22}}+J_{33} \frac{\partial \phi}{\partial \eta_{32}} \\
& T_{33}=J_{31} \frac{\partial \phi}{\partial \eta_{13}}+J_{32} \frac{\partial \phi}{\partial \eta_{23}}+J_{33} \frac{\partial \phi}{\partial \eta_{33}}
\end{aligned}
$$

where $J_{i j}$ are the Jacobian matrix elements, $\eta_{i j}$ are the strain matrix elements, and $\phi$ is the elastic potential energy.

$J_{i j}$ is determined using $x=a+u, y=b+v$, and $z=$ $c+w$. According to the acoustoelasticity theory, the transverse and longitudinal ultrasonic wave propagation direction and the polarization direction are related to the stress direction as follows [14]:

(1) The propagation of a longitudinal wave along the direction of stress is given by

$$
\begin{aligned}
\rho_{0} V_{111}^{2}= & \lambda+2 \mu \\
& +\frac{\sigma}{3 K_{0}}\left[\frac{\lambda+\mu}{m}(4 \lambda+10 \mu+4 m)+\lambda+2 l\right] .
\end{aligned}
$$

(2) The stress direction along the propagation direction, with the polarization direction perpendicular to the shear stress, is given as

$$
\rho_{0} V_{113}^{2}=\lambda+2 \mu+\frac{\sigma}{3 K_{0}}\left[2 l-\frac{2 \lambda}{u}(\lambda+2 \mu+m)\right],
$$

where for $V_{i j k} i$ is the direction of wave propagation, $j$ is the wave polarization direction, and $k$ is the load direction. In (5) and (6), the constant $K_{0}$ is given by

$$
K_{0}=\lambda+\frac{2}{3} \mu
$$

$\rho_{0}$ is the density and $\lambda$ and $\mu$ and $l, m$, and $n$ are second- and third-order elastic constants of the material, respectively. For the purposes of a semi-infinite plate,

$$
\begin{aligned}
& \lambda=\rho_{0} V_{111}^{2}-2 \mu, \\
& \mu=\rho_{0} V_{131}^{2} .
\end{aligned}
$$

According to the basic assumptions of elasticity, before the axial stress, less than the yield limit of the bolt, the material can be considered as a complete bolt steel elastomer. Thus, when measuring the temperature constant at room temperature, the second bolt material and the third-order elastic constants do not contribute to increases or changes in the axial stress. Under these conditions, the only factor that has an effect on the axial bolt stress and bolt length measurement is the measurement temperature. Considering 
that the effective stress which can tighten the nut bolt deputy district is not wrapped in the entire area, as shown in Figure 1, we can create a physical model of the axial stress during fastening of the bolt $[8,9]$. The head of the bolt is placed in transverse and longitudinal wave transducers. The pulse echo method is used to measure the horizontal and vertical wave crossings in the screw. The bolt axial stress and length measurement can be obtained indirectly from these measurements.

Taking into account the measured temperature expansion of the overall impact of the bolt and the linear elastic deformation zone length for the bolt stress, the bolt length can be expressed as follows [15]:

$$
\begin{aligned}
L & =(1+\Delta t \beta) l, \\
L^{\prime} & =\left(1+\frac{\sigma}{E}\right) l^{\prime}, \\
\Delta t & =t-t_{0}, \\
V_{L_{t}} & =V_{L_{0}}\left(1-\alpha_{L} \Delta t\right), \\
V_{S_{t}} & =V_{S_{0}}\left(1-\alpha_{S} \Delta t\right) ;
\end{aligned}
$$

$L$ is the length of the bolt and $L^{\prime}$ is the length of the stress region, $l$ is the length of the bolt and $l^{\prime}$ is the length of the stress region which is not influenced by temperature, $t$ and $t_{0}$ are the current temperature and standard temperature $\left(26^{\circ} \mathrm{C}\right), V_{L_{t}}$ and $V_{S_{t}}$ are the velocity of longitudinal wave and transverse wave at temperature of $t^{\circ} \mathrm{C}, \beta$ is the linear thermal expansion coefficient, $\alpha_{L}$ and $\alpha_{S}$ are the sound velocity coefficient of longitudinal wave and transverse wave with the change of temperature, and $E$ is Young's modulus.

The relationship between the axial shear stress on the bolt and the longitudinal wave propagation velocity can be expressed as

$$
\begin{aligned}
& \rho_{0}\left[\frac{2 L^{\prime}(1+\Delta t \beta)(1+\sigma / E)}{T_{L}-2\left(L-L^{\prime}\right)(1+\Delta t \beta) / V_{L_{0}}\left(1-\alpha_{L} \Delta t\right)}\right]^{2} \\
& =\lambda+2 \mu \\
& \quad+\frac{\sigma}{3 K_{0}}\left[\frac{\lambda+\mu}{m}(4 \lambda+10 \mu+4 m)+\lambda+2 l\right], \\
& \rho_{0}\left[\frac{2 L^{\prime}(1+\Delta t \beta)(1+\sigma / E)}{T_{S}-2\left(L-L^{\prime}\right)(1+\Delta t \beta) / V_{S_{0}\left(1-\alpha_{S} \Delta t\right)}}\right]^{2} \\
& =\mu+\frac{\sigma}{3 K_{0}}\left[\frac{\lambda n}{m}+4 \lambda+4 \mu+m\right] .
\end{aligned}
$$

Considering that the transit time of longitudinal wave and transverse wave is very short, the experiment is performed at indoor temperature, and the order of magnitudes of $\alpha_{L}$ and $\alpha_{S}$ is $10^{-4}$, the change in temperature can be neglected. The original equation can be simplified to

$$
\frac{\left(T_{S}-2 A / V_{S_{0}}\right)^{2}}{\left(T_{L}-2 A / V_{L_{0}}\right)^{2}}=\frac{\lambda+2 \mu+\left(K_{L} / 3 K_{0}\right) \sigma}{\mu+\left(K_{S} / 3 K_{0}\right) \sigma}
$$

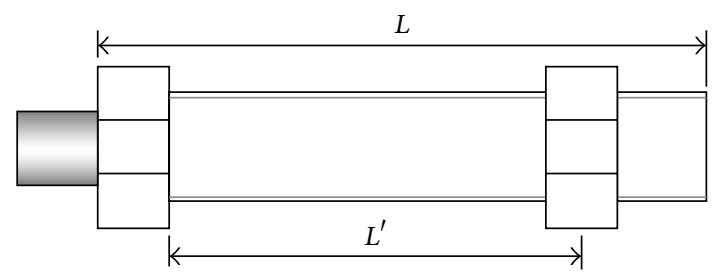

FIGURE 1: Bolt axial stress measurement physical model.

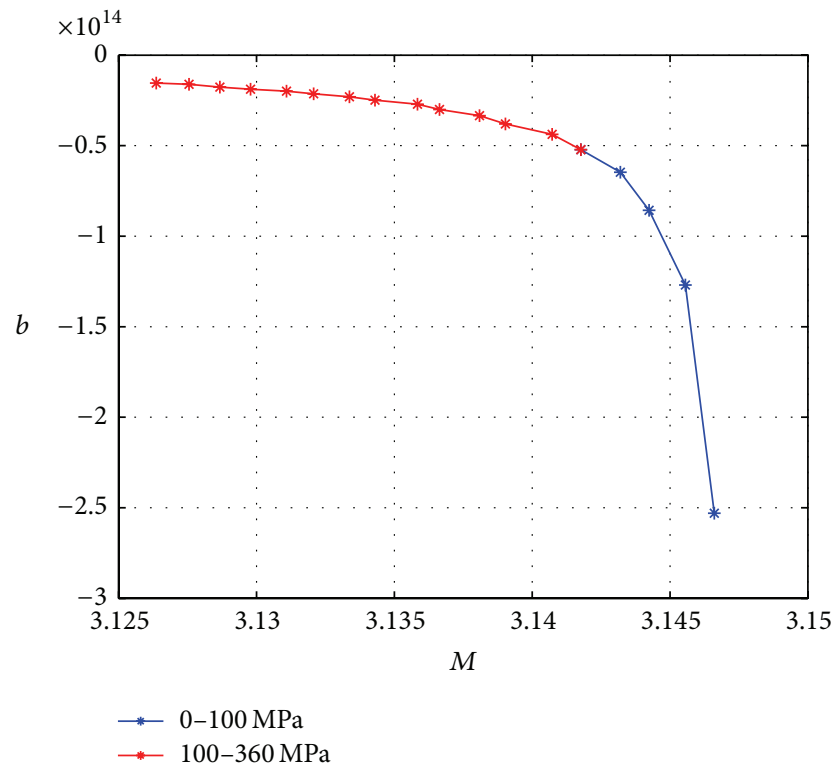

FIGURE 2: The fitting curve $M-b$ of an A2-70 austenitic stainless steel bolt.

To simplify further, we define $M$ as the left side of (11):

$$
M=\frac{\left(T_{S}-2 A / V_{S_{0}}\right)^{2}}{\left(T_{L}-2 A / V_{L_{0}}\right)^{2}},
$$

where $T_{L}$ is the time of propagation of longitudinal wave and $T_{S}$ is the time of propagation of shear wave.

The final expression for the stress $\sigma$ is given by

$$
\sigma=\frac{(M-2) \mu-\lambda}{K_{L} / 3 K_{0}-M K_{S} / 3 K_{0}} .
$$

The two coefficients in (14), $K_{S}$ and $K_{L}$, can be obtained via an axial tensile test:

$$
K_{L}=M K_{S}+3 K_{0} \frac{(M-2) \mu-\lambda}{\sigma} .
$$

To facilitate the calculation, we define $b$ as the left side of

$$
b=3 K_{0} \frac{(M-2) \mu-\lambda}{\sigma} .
$$


TABLE 1: Austenitic stainless steel bolt (A2-70) axial tensile stress measurement results.

\begin{tabular}{lcccc}
\hline Stress & $T_{L}(\mu \mathrm{s})$ & $T_{S}(\mu \mathrm{s})$ & $M$ & $b$ \\
\hline 0 & 42.831 & 76.17 & 3.145160 & \\
20 & 42.8415 & 76.204 & 3.146601 & $-2.53 \times 10^{14}$ \\
40 & 42.8605 & 76.2265 & 3.145552 & $-1.27 \times 10^{14}$ \\
60 & 42.88 & 76.247 & 3.14423 & $-8.57 \times 10^{13}$ \\
80 & 42.897 & 76.266 & 3.143192 & $-6.47 \times 10^{13}$ \\
100 & 42.9155 & 76.2835 & 3.14176 & $-5.22 \times 10^{13}$ \\
120 & 42.9325 & 76.3025 & 3.140722 & $-4.38 \times 10^{13}$ \\
140 & 42.9515 & 76.318 & 3.13902 & $-3.80 \times 10^{13}$ \\
160 & 42.9685 & 76.338 & 3.13808 & $-3.34 \times 10^{13}$ \\
180 & 42.988 & 76.357 & 3.13663 & $-3.00 \times 10^{13}$ \\
200 & 43.0044 & 76.3775 & 3.13583 & $-2.71 \times 10^{13}$ \\
220 & 43.0245 & 76.3965 & 3.13429 & $-2.49 \times 10^{13}$ \\
240 & 43.042 & 76.4175 & 3.13336 & $-2.30 \times 10^{13}$ \\
260 & 43.062 & 76.439 & 3.13207 & $-2.14 \times 10^{13}$ \\
280 & 43.081 & 76.462 & 3.131084202 & $-1.99 E+13$ \\
300 & 43.1025 & 76.486 & 3.129780475 & $-1.88 E+13$ \\
320 & 43.124 & 76.512 & 3.128663993 & $-1.77 E+13$ \\
340 & 43.148 & 76.5355 & 3.127547129 & $-1.61 E+13$ \\
360 & 43.1755 & 76.5565 & 3.12635963 & $-1.54 E+13$ \\
\hline
\end{tabular}

$V_{L_{0}}=5638.499 \mathrm{~m} \mathrm{~s}^{-1}$.

$V_{S_{0}}=3105.499 \mathrm{~m} \mathrm{~s}^{-1}$.

$\lambda=9.8160 \times 10^{10}$.

$\mu=7.5710 \times 10^{10}$.

$\rho=7850 \mathrm{~kg} \mathrm{~m}^{-3}$.

Explanation:

Stress [MPa] bolt axial stress.

$T_{L}[\mathrm{~s}]$ time of propagation of longitudinal wave.

$T_{S}[\mathrm{~s}]$ time of propagation of shear wave.

$\lambda \mu$ the second-order elastic constants of the material.

\section{Experiments and Results}

The experimental measurement system consisted of a symmetrical configuration of transverse and longitudinal wave transducers. The transverse and longitudinal wave transducer units operated independently and simultaneously produced transverse and longitudinal wave excitation signals (center frequency: $2.25 \mathrm{MHz}$ ). The broadband contact transducer produced a narrow pulse (diameter: $10 \mathrm{~mm}$ ). The bolts whose models are M20 include A2-70, 4.8, and 8.8. In order to achieve stable coupling effects, nuts and flat tail had grinded the grips smooth, and the temperature is controlled at $26^{\circ} \mathrm{C}$.

The system used an ultrasonic excitation pulse signal transceiver device. Oscilloscopes coupled to the transverse and longitudinal wave transducer units provided an accurate recording of the transit time. A stretching machine was used to quantify the tensile test results. The stress value was extrapolated from the time duration through the function relationship between the two. Due to the low yield strength of steel (maximum bolt strength: $320 \mathrm{MPa}$ ), the tensile test stress range for the A2-70 bolts was $0-360 \mathrm{MPa}$, using a step increment of $20 \mathrm{MPa}$; the axial stress range for the lowcarbon steel 4.8 and 8.8 bolts was $0-260 \mathrm{MPa}$, in $20 \mathrm{MPa}$ step
TABLE 2: Tensile stress measurement results for a low-carbon 4.8 steel bolt.

\begin{tabular}{lcccc}
\hline Stress & $T_{L}(\mu \mathrm{s})$ & $T_{S}(\mu \mathrm{s})$ & $M$ & $b$ \\
\hline 0 & 46.3535 & 84.2395 & 3.27610 & \\
20 & 46.369 & 84.256 & 3.27509 & $-5.27 \times 10^{14}$ \\
40 & 46.3865 & 84.274 & 3.273907 & $-2.65 \times 10^{14}$ \\
60 & 46.405 & 84.29 & 3.272383 & $-1.78 \times 10^{13}$ \\
80 & 46.424 & 84.3155 & 3.27161 & $-1.34 \times 10^{13}$ \\
100 & 46.44 & 84.3375 & 3.271006 & $-1.07 \times 10^{13}$ \\
120 & 46.458 & 84.358 & 3.26995 & $-8.96 \times 10^{13}$ \\
140 & 46.4755 & 84.382 & 3.26929 & $-7.70 \times 10^{13}$ \\
160 & 46.4945 & 84.3985 & 3.26774 & $-6.78 \times 10^{13}$ \\
180 & 46.513 & 84.417 & 3.26644 & $-6.05 \times 10^{13}$ \\
200 & 46.533 & 84.432 & 3.26460 & $-5.49 \times 10^{13}$ \\
220 & 46.555 & 84.456 & 3.2632 & $-5.01 \times 10^{13}$ \\
240 & 46.575 & 84.4745 & 3.26171 & $-4.68 \times 10^{13}$ \\
260 & 46.6 & 84.5 & 3.26001 & $-4.39 \times 10^{13}$ \\
\hline$V_{L_{0}}=5658.406 \mathrm{~m} \mathrm{~s}^{-1} \cdot$ & & & \\
$V_{S_{0}}=3150.549 \mathrm{~m} \mathrm{~s}^{-1}$. & & & \\
$\lambda=12.087 \times 10^{10} \cdot$ & & & \\
$\mu=7.8910 \times 10^{10} \cdot$ & & & \\
$\rho=7850 \mathrm{~kg} \mathrm{~m}^{-3} \cdot$ & & &
\end{tabular}

TABLE 3: Axial tensile stress measurement results for a low-carbon 8.8 steel bolt.

\begin{tabular}{lcccc}
\hline Stress & $T_{L}(\mu \mathrm{s})$ & $T_{S}(\mu \mathrm{s})$ & $M$ & $b$ \\
\hline 0 & 46.402 & 84.484 & 3.28979 & \\
20 & 46.412 & 84.4885 & 3.2886 & $-4.99 \times 10^{14}$ \\
40 & 46.427 & 84.495 & 3.286792 & $-2.51 \times 10^{14}$ \\
60 & 46.4405 & 84.505 & 3.285529 & $-1.69 \times 10^{14}$ \\
80 & 46.456 & 84.514 & 3.283863 & $-1.27 \times 10^{14}$ \\
100 & 46.47 & 84.524 & 3.282523 & $-1.02 \times 10^{14}$ \\
120 & 46.487 & 84.533 & 3.280624 & $-8.59 \times 10^{13}$ \\
140 & 46.5025 & 84.543 & 3.27905 & $-7.41 \times 10^{13}$ \\
160 & 46.519 & 84.552 & 3.277234 & $-6.53 \times 10^{13}$ \\
180 & 46.5335 & 84.562 & 3.275821 & $-5.84 \times 10^{13}$ \\
200 & 46.549 & 84.572 & 3.274252 & $-5.29 \times 10^{13}$ \\
220 & 46.57 & 84.582 & 3.27182 & $-4.85 \times 10^{13}$ \\
240 & 46.5825 & 84.59 & 3.270553 & $-4.47 \times 10^{13}$ \\
260 & 46.6 & 84.6 & 3.268675 & $-4.16 \times 10^{13}$ \\
\hline
\end{tabular}

$V_{L_{0}}=5958.466 \mathrm{~m} \mathrm{~s}^{-1}$.

$V_{S_{0}}=3170.589 \mathrm{~m} \mathrm{~s}^{-1}$.

$\lambda=12.087 \times 10^{10}$.

$\mu=7.8910 \times 10^{10}$.

increments. Tables 1-3 show the axial stress measurement results for the three bolt types.

The $b-M$ trend curves and comparison results of the three materials, A2-70, 4.8, and 8.8, are shown in Figures 2-7, respectively.

The distribution curves shown in Figures 2, 4, and 6 were used to determine the stress coefficients, $K_{S}$ and $K_{L}$, from fitting of the data. From the $b-M$ graphs, two linear stress regions were identified: $0-100$ and $100-360 \mathrm{MPa}$. This 
TABLE 4: Matlab least squares fit of the stress measurement coefficients, $K_{S}$ and $K_{L}$.

\begin{tabular}{lcccc}
\hline \multirow{2}{*}{ Material } & \multicolumn{4}{c}{ Coefficient } \\
& $K_{S}$ & $K_{L}$ & $K_{S}$ & $K_{L}$ \\
\hline A2-70 & $3.7835 \times 10^{16}$ & $1.1885 \times 10^{17}$ & $2.813 \times 10^{15}$ & $8.7741 \times 10^{15}$ \\
4.8 & $5.5036 \times 10^{16}$ & $1.7992 \times 10^{17}$ & $4.2938 \times 10^{15}$ & $1.396 \times 10^{16}$ \\
8.8 & $3.3484 \times 10^{16}$ & $1.0982 \times 10^{17}$ & $3.543 \times 10^{15}$ & $1.1544 \times 10^{16}$ \\
\hline
\end{tabular}

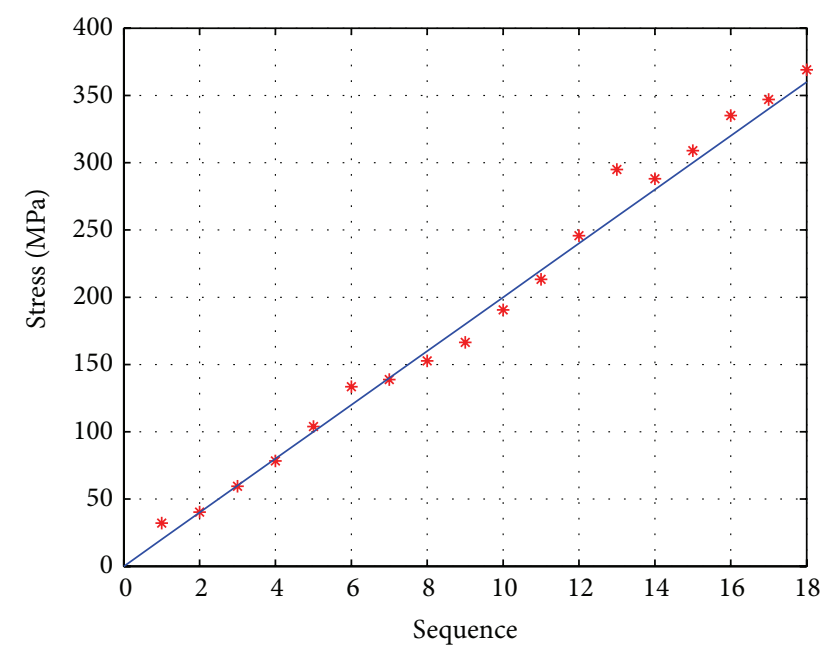

Figure 3: The comparison result of an A2-70 austenitic stainless steel bolt.

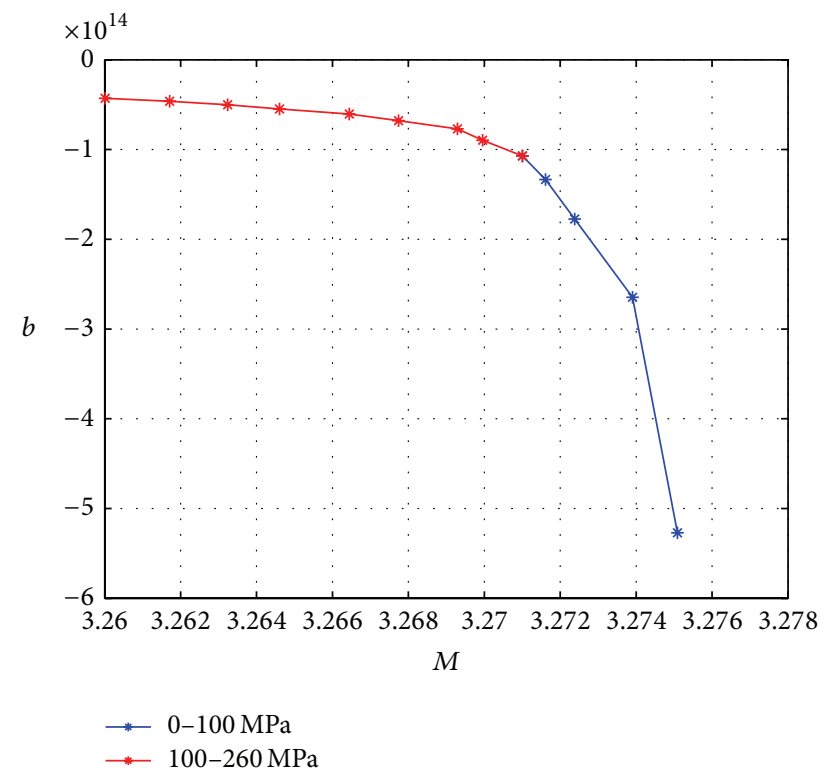

Figure 4: The fitting curve $M-b$ of a 4.8 low-carbon steel bolt.

is mainly because the screw thread pair of bolts generates quoin, friction, and elastic deformation of screw thread pair at first $(0-100 \mathrm{MPa})$ and then enters into the elastic stage $(100-360 \mathrm{MPa})$. The corresponding $K_{S}$ and $K_{L}$ values of the three bolt types were determined from a least squares fit using Matlab software. Table 4 shows the $K_{S}$ and $K_{L}$ values for the

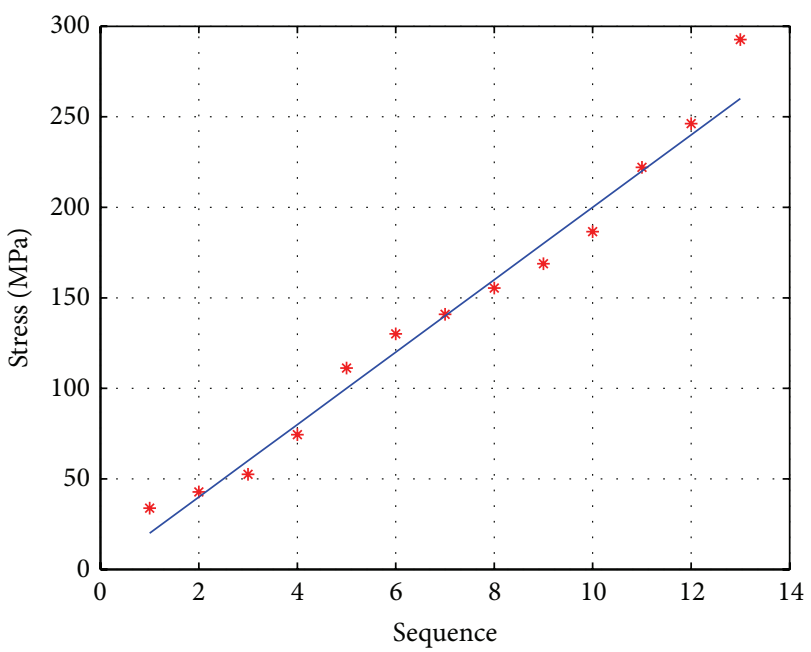

FIGURE 5: The comparison result of a 4.8 low-carbon steel bolt.

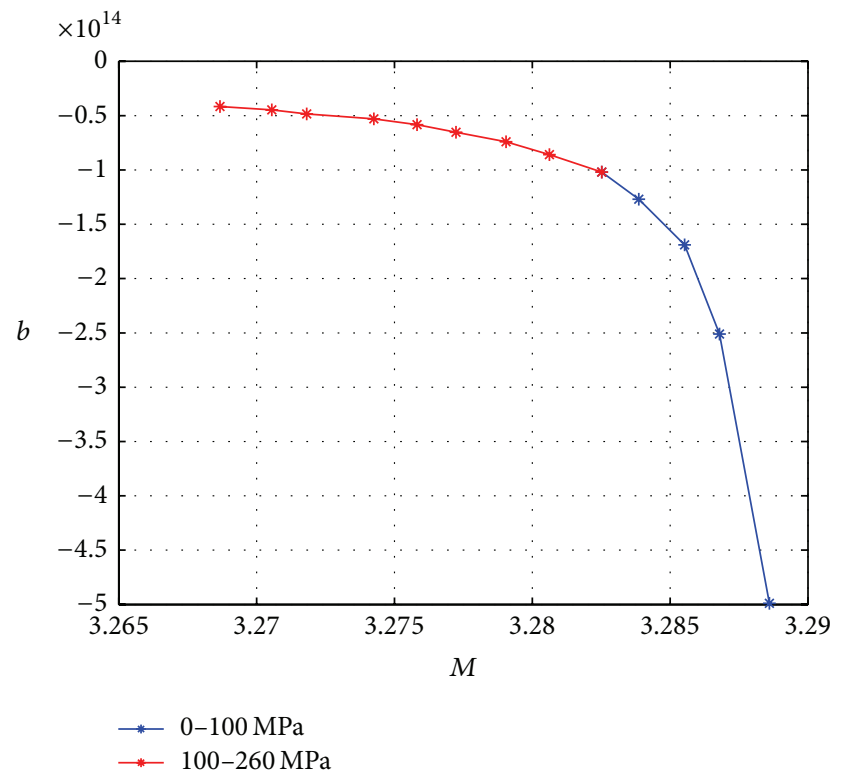

Figure 6: The fitting curve $M-b$ of a 8.8 low-carbon steel bolt.

three stress measurement regions. The average error of this new method is less than $20 \mathrm{MPa}$ from Figures 3, 5, and 7.

\section{Conclusions}

In this paper, an ultrasonic technique involving the combination of transverse and longitudinal waves provided a fast and effective way to measure bolt axial stress in three bolt materials: an austenitic stainless steel bolt (A2-70) and low-carbon steel 4.8 and 8.8 bolts. In the measurement process, the effect of temperature and elastic deformation was eliminated allowing the axial length of the bolt and the plug axial stress to be measured. The measurements were performed under various load conditions. The stress measurement coefficients, determined from least squares fitting of the stress data, were classified into two stress ranges, 


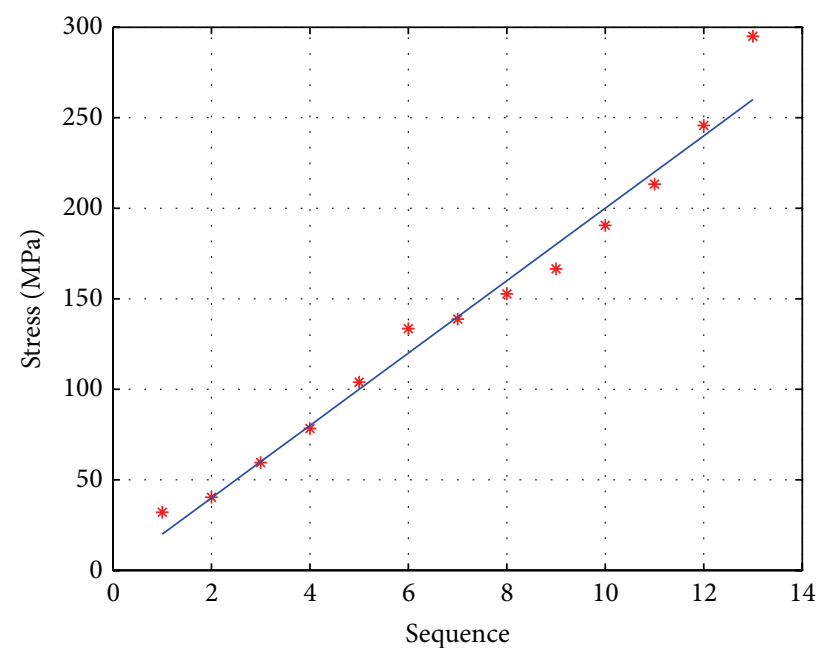

FIGURE 7: The comparison result of a 8.8 low-carbon steel bolt.

to provide a more accurate reading of the bolt axial stress. The average error of this new method is less than $20 \mathrm{MPa}$ and can be applied to various industries, including aerospace, marine, turbines, bridge construction, chemical equipment, and energy systems.

\section{Conflict of Interests}

The authors declare that there is no conflict of interests regarding the publication of this paper.

\section{Acknowledgments}

This project is supported by the National Natural Science Foundation of China (Grant no. 51305028) and Beijing Higher Education Young Elite Teacher Work.

\section{References}

[1] K. Maruyama, "Stress analysis of a bolt-nut joint by the finite element method and the copper-electroplating method," Bulletin of JSME, vol. 17, no. 106, pp. 442-450, 1974.

[2] H. D. Williams, D. Armstrong, and R. H. Robins, "Application of ultrasonic stress measurements to problems in the electricity supply industry," Journal of Testing and Evaluation, vol. 10, no. 5, pp. 217-222, 1982.

[3] G. C. Johnson, A. C. Holt, and B. Cunningham, "An ultrasonic method for determining axial stress in bolts," Journal of Testing and Evaluation, vol. 14, no. 5, pp. 253-259, 1986.

[4] A. J. Koshti, "Preload measurement in sleeve bolts using an ultrasonic technique," Materials Evaluation, vol. 54, no. 2, pp. 308-313, 1996.

[5] T. Guan, "Analysis of high-strength fasteners fastening," Equipment Manufacturing Technology, vol. 4, pp. 163-171, 2009.

[6] J. Deputat, "Ultrasonic technique for measuring stress in screw," Solid State Technology, 1979.

[7] E. Tanala, G. Bourse, M. Fremiot, and J. F. De Belleval, "Determination of near surface residual stresses on welded joints using ultrasonic methods," NDT \& E International, vol. 28, no. 2, pp. 83-88, 1995.

[8] J. L. Rose, Ultrasonic Waves in Solid Media, Cambridge University Press, 2004-2009.

[9] G. Yang, F. Wang, J. Hong, and L. Zhu, "Theoretical analysis for bolted member stiffness," Journal of Xian Jiaotong University, vol. 46, no. 7, pp. 50-56, 2012.

[10] S. A. Nassar and A. Abboud, "An improved stiffness model for bolted joints," Journal of Mechanical Design, vol. 131, no. 12, Article ID 121001, 11 pages, 2009.

[11] J. C. Musto and N. R. Konkle, "Computation of member stiffness in the design of bolted joints," Journal of Mechanical Design, vol. 128, no. 6, pp. 1357-1360, 2006.

[12] N. Chandrasekaran and K. Salamu, Relationship Between Stress and Temperature Dependence of Ultrasonic Shear Velocity, Plenum Press, New York, NY, USA, 1984.

[13] C. O. Ruud and R. E. Green Jr., Nondestructive Methods for Material Property Determination, Plenum Press, 1984.

[14] J. L. Rose, Ultrasonic Waves in Solid Media, Cambridge University Press, Cambridge, UK, 1999.

[15] Z. Jiang, "The influence of temperature on the ultrasonic wave velocity and stress measurement," Nondestructive Testing, vol. 6, 1999. 

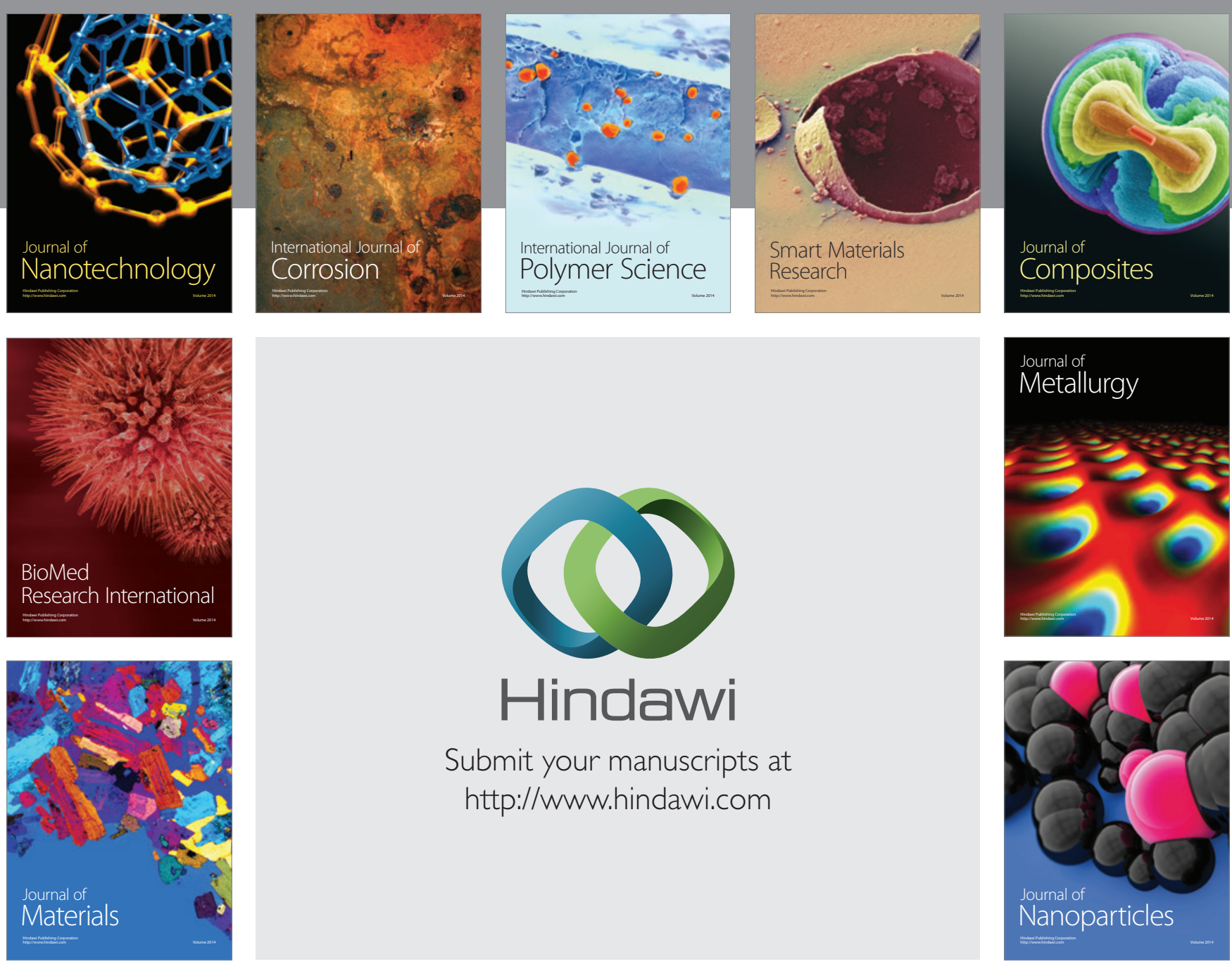

Submit your manuscripts at http://www.hindawi.com
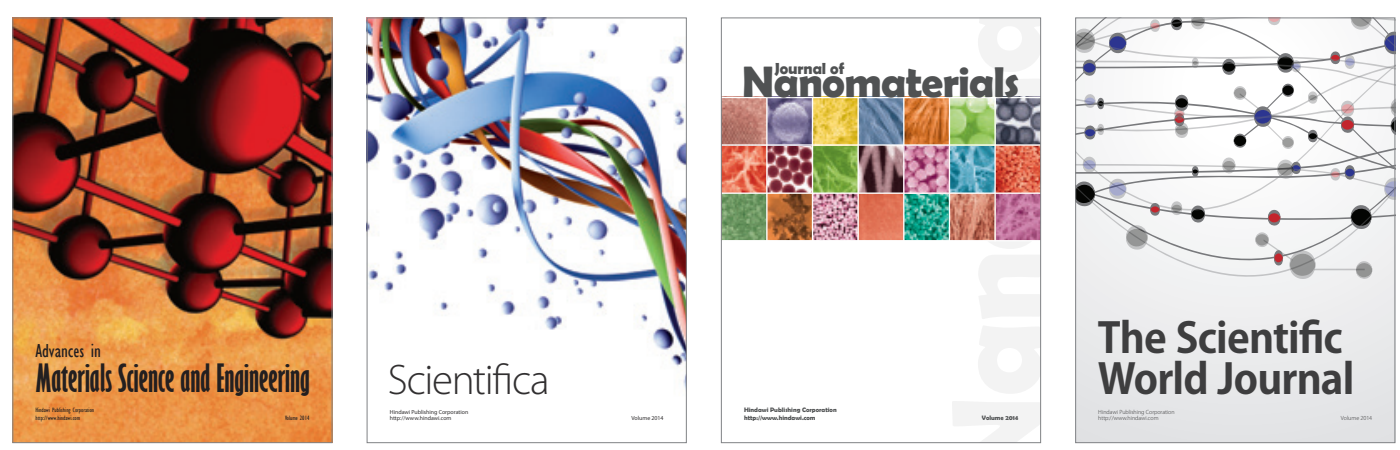

\section{The Scientific World Journal}
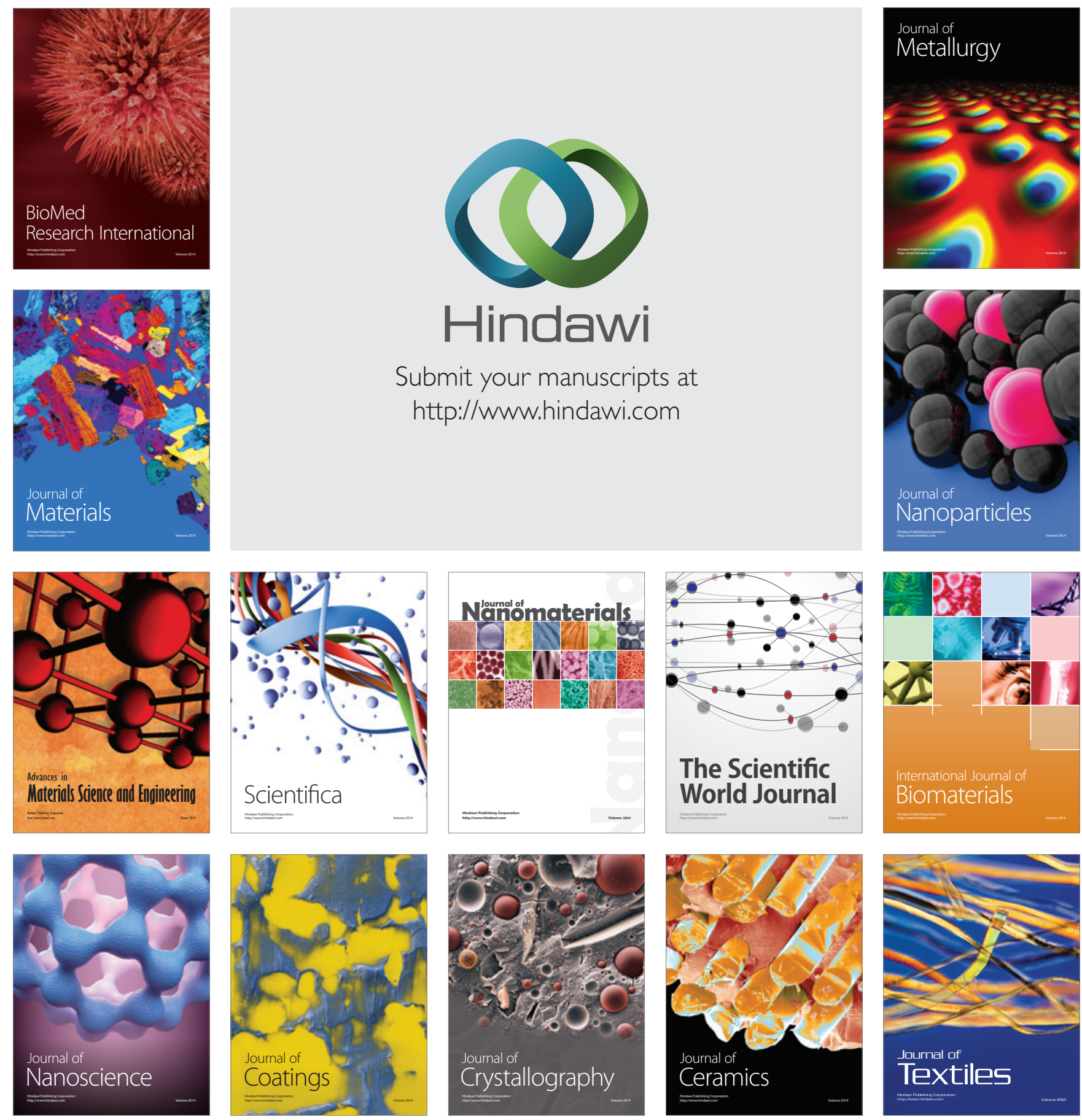